\title{
A novel mutation in the DNMT1 gene in a patient presenting with pure cerebellar ataxia
}

\author{
Hussein Algahtani ${ }^{1} *$ and Bader Shirah ${ }^{2}$ \\ ${ }^{1}$ Neurology Section, Department of Medicine, King Abdulaziz Medical City, King Saud bin Abdulaziz University for Health Sciences, Jeddah, \\ Saudi Arabia \\ ${ }^{2}$ King Abdullah International Medical Research Center, King Saud bin Abdulaziz University for Health Sciences, Jeddah, Saudi Arabia
}

\begin{abstract}
Mutations in the DNA methyltransferase 1 gene (DNMT1) were reported to cause two phenotypes: OMIM 604121 and OMIM 614116. The first phenotype includes autosomal dominant cerebellar ataxia, deafness, and narcolepsy, which were reported to be caused by mutations in exon 21 . The second phenotype includes hereditary sensory and autonomic neuropathy type $1 \mathrm{E}$, which was suggested to be caused by mutations in exon 20 and 21 . In this article, we report a novel heterozygous missense variant c.898A>C, p.(Lys300GIn) in exon 12 of DNMT1 in a young woman who presented with pure cerebellar ataxia. This report indicates that a mutation in exon 12 may lead to pure cerebellar ataxia. Another possibility is that the patient is currently in an early stage of the disease, and as the disease progresses, she will have more manifestations. To confirm or exclude this possibility, a subsequent follow-up study reporting the disease progression in this patient may be needed. Further reports of cases with the same mutation are needed to confirm the phenotype of this mutation.
\end{abstract}

Key words: DNMT1, DNA methylation, Autosomal dominant cerebellar ataxia, Novel mutation, Saudi Arabia.

\section{Introduction}

The inherited ataxias are a clinically and genetically heterogeneous group of diseases that occur due to dysfunction of the cerebellum and its connections [1]. In most of these disorders, the disease onset is between 30 and 50 years of age. Several mutations in a variety of genes have been identified to cause these ataxias [2]. For instance, mutations in the DNA methyltransferase 1 gene (DNMT1) were reported to cause two phenotypes: OMIM 604121 and OMIM 614116. The first phenotype includes autosomal dominant cerebellar ataxia, deafness, and narcolepsy, which were reported to be caused by mutations in exon 21 . The second phenotype includes hereditary sensory and auto- nomic neuropathy type $1 \mathrm{E}$, which was reported to be caused by mutations in exon 20 and $21[3,4]$. In this article, we report a novel mutation in exon 12 of DNMT1 in a young woman who presented with pure cerebellar ataxia. This report expands the heterogeneity of the clinical presentation of DNMT1-associated diseases.

\section{Case}

A 30-year-old Saudi woman, a mother of two children, presented with a 6-year history of gradually progressive ataxia, imbalance, and frequent falls. Over the last year, she has had great difficulties performing activities of daily living, including taking

\footnotetext{
Received: 9 June 2017, Revised: 18 July 2017, Accepted: 9 September 2017, Published: 31 December 2017

*Corresponding author: Hussein Algahtani, M.D., FRCPC

Neurology Section, Department of Medicine, King Abdulaziz Medical City, King Saud bin Abdulaziz University for Health Sciences, P.0. Box: 12723, Jeddah 21483, Saudi Arabia.

Tel: +966-556633130, E-mail: halgahtani@hotmail.com

Conflict of interest: The authors declare that they do not have any conflicts of interest.

(c) This is an open-access article distributed under the terms of the Creative Commons Attribution Non-Commercial License (http://creativecommons.org/licenses/by-nc/4.0/) which permits unrestricted non-commercial use, distribution, and reproduction in any medium, provided the original work is properly cited.

(c) Copyright 2017 by the Korean Society of Medical Genetics and Genomics www.e-kjgm.org
} 
care of her children and cooking. There was no history of fever, joint pain, skin rash, visual symptoms, mouth or genital ulcers, or gastrointestinal symptoms. The patient denied memory loss or psychiatric symptoms. Her past medical history was unremarkable, including endocrine disorders, infections, sleep disorders, or trauma. Family history was negative for a similar condition, but her parents are first-degree relatives. On examination, the patient was conscious and oriented with normal cognitive functions. Her reflexes were brisk, and she had bilateral downgoing toes. She was dysarthric with cerebellar ataxic speech, and she had horizontal nystagmus. The rest of her cranial nerve examination was normal. Motor examination showed a normal tone and power. Cerebellar examination showed dysmetria and dysdiadokinesia. She had difficulty walking without assistance. There were no associated skeletal deformities, and her fundus examination was normal. Examination of her gait showed a wide-based gait with a tendency to fall to either side. Extensive blood work including both routine and specific tests to rule out the cause of ataxia in this age group was negative. These include the level of vitamin $E$, the level of vitamin B-12, a blood smear for acanthocytes, viral serology, a thyroid function test, serology for celiac disease, and lipoprotein electrophoresis. Magnetic resonance imaging (MRI) of the brain revealed symmetrical cerebellar atrophy with mild generalized brain atrophy (Fig. 1). Cerebrospinal fluid analysis revealed a normal cell count and biochemical profile with negative oligoclonal bands. Visual evoked potential, visual field perimetry, and nerve conduction studies were normal. Family and genetic counseling were offered through a genetic consultant.

The patient visited the clinic and blood samples were collected and sent for genetic testing after obtaining informed written consent according to the Declaration of Helsinki. Total genomic DNA was extracted from the biological sample. DNA quality and quantity were assessed using a fluorometric electrophoresis method. Extracted total genomic DNA was mechanically fragmented and enzymatically end-repaired. DNA adapters were added using a ligation-based method and the sequencing library was amplified using polymerase chain reaction (PCR). The quality and quantity of the sequencing library DNA was assessed through electrophoresis and fluorometric analyses, respectively. A proprietary oligonucleotide-selective sequencing method was used for capturing genomic targets and sequencing was performed using an Illumina sequencing system (Illumina, San Diego, CA, USA).

The Blueprint Genetics Ataxia Panel was used to analyze gene sequences that are associated with cerebellar ataxia, episodic ataxia, and spinocerebellar ataxia. It is composed of 141 genes $(2,196$ exons) with a median coverage of 241 . The panel targets all protein coding exons and the exon-intron boundaries of all target genes. It also covers a number of mutations located outside the coding regions of these genes. This diagnostic tool covers the majority of cerebellar ataxia, episodic ataxia, and spinocerebellar ataxia mutations known to date.

Raw sequence reads were filtered to exclude reads with ambiguous base calls and trimmed from the 3 ' ends based on base call quality and the presence of the adapter, poly-A, or capture oligo sequence. The remaining high-quality reads were mapped to the human genome reference sequence ( $\mathrm{Hg} 19)$. Single nucleotide variants and short insertions and deletions were identified using a proprietary data analysis pipeline. The pathogenicity of the identified variants was predicted based on the biochemical properties of the codon change and the degree of evolutionary conservation using PolyPhen, SIF, and Mutation Tester. Identified variants were annotated using allelic frequencies from large population studies.

The pathogenicity of single nucleotide variants and short in-

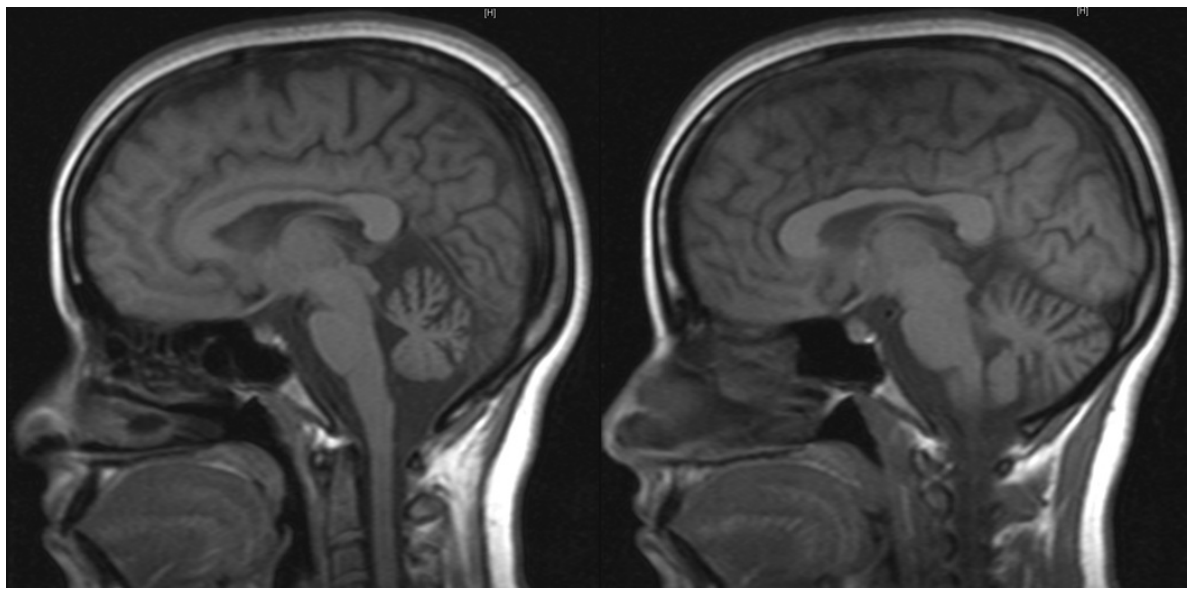

Fig. 1. Magnetic resonance imaging of the brain showing symmetrical cerebellar atrophy with mild generalized brain atrophy. 
sertion and deletion variants was assessed by evaluating allele frequency, in silico predictions, and the annotations from public variant databases and related medical literature. Novel single nucleotide variants, and short insertion and deletion variants classified as pathogenic or likely pathogenic, as well as variants of uncertain significance with a quality score less than 500 were confirmed using direct Sanger sequencing of the PCR amplicons. Confirmation of recurrent pathogenic or likely pathogenic variants was initially performed for three consequent cases using Sanger sequencing, and subsequently only when variant quality was required. This study was approved by the Institutional Review Board of King Abdullah International Medical Research Center.

The sequence analysis identified a heterozygous missense variant c.898A>C, p.(Lys300GIn) in exon 12/41 of DNMT1, which is inherited in an autosomal dominant manner. This variant has not been reported in the public mutation databases or in the literature. It is classified as a variant of uncertain significance, according to the ACMG guidelines. The variant is predicted benign by in silico pathogenicity prediction tools. In addition, she was a heterozygous carrier for three missense novel mutations in the MARS2 (c.1094A>G, p.(Tyr365Cys)), GBA2 (c.2201G>A, p.(Arg734His)), and ABHD12 (c.62C >T, p.(Ser21Phe)) genes. Since the diseases caused by mutations in these genes are inherited in an autosomal recessive manner and the patient is heterozygous for the variants, it is less likely that they would be the cause of her present phenotype.

\section{Discussion}

The DNMT1 gene is located on chromosome 19p13.2 and is responsible for encoding the enzyme DNA methyltransferase 1 , which is involved in maintaining the patterns of methylated cytosine residues in the human genome. DNA methylation is a key process for gene expression owing to its effect on DNA replication and repair [5] (Fig. 2). DNMT1 is expressed in several tissues and developmental stages. DNMT1 is active in the nervous system, but its exact function is not thoroughly understood. It is suggested to play a role in regulating neuron maturation, differentiation, migration, and survival. Although DNMT1 is expressed in a variety of tissues, mutations in this gene have been linked only with neurodegenerative diseases for an unknown reason. This suggests that DNMT1 has a specific mechanism for dynamic regulation and neuronal survival [6].

A recently published study investigating mutations in exon 20 found that these mutations affect DNMT1 interaction with the essential cofactor UHRF1 (ubiquitin-like with PHD and ring finger domains 1), which is believed to cause cell cycle-dependent degradation of DNMT1 and lead to impaired neuronal differentiation [7]. Further studies reporting different cohorts with a variety of mutations in DNMT1 need to be published to further understand the pathogenic mechanism. In addition, the methylation status of these patients has recently been studied, and it was reported that patients with DNMT1 mutations have similar hypomethylation at repetitive elements and hypermethylation
(A)

\begin{tabular}{|lllll|}
\hline Regulatory domain & \multicolumn{2}{c}{ Catalytic domain } \\
\cline { 2 - 3 } & CXXC & BAH1 & BAH 2 & MTase \\
\hline
\end{tabular}

DNMT 1

(B)

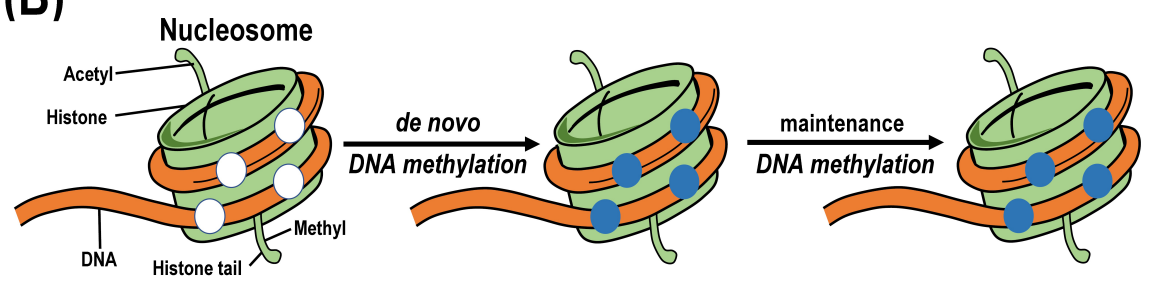

Fig. 2. Schematic representation of $(A)$ the structure of DNMT1 gene and (B) the process of DNA methylation. 
at specific gene/promoter/CpG islands, which is an important finding to expand the understanding of the molecular pathogenesis [8].

Heterozygous mutations in exon 21 of DNMT1 have been identified in patients with adult onset cerebellar ataxia, narcolepsy/cataplexy, sensorineural deafness, and dementia, and features that are more variable include optic atrophy, sensory neuropathy, psychosis, and depression [9]. Heterozygous mutations in exon 20 and 21 of DNMT1 have been identified in patients with adult onset progressive peripheral sensory loss associated with progressive hearing impairment and early-onset dementia $[4,10]$. The disease penetrance is estimated to be high. Nineteen disease-causing variants were reported in exons 20 and 21, including 15 missense variants, two small deletions, one small insertion, and one small insertion-deletion. These mutations affect the replication foci targeting sequence domains that are responsible for regulating the binding of DNMT1 to chromatin [11].

History and physical examination are of paramount importance in indicating a diagnosis of cerebellar ataxia. MRI of the brain is important in evaluating structural abnormalities such as cerebellar atrophy. Genetic testing remains the only definitive diagnostic method to identify the responsible gene. Management of these patients mainly includes occupational and physical therapy for gait dysfunction and speech therapy. There is no currently available treatment that can cure the disease or alter disease progression. Prognosis is variable in these patients but improvement in their condition is unlikely [1-3].

In conclusion, we reported a novel heterozygous missense variant c.898A $>$ C, p.(Lys300GIn) in exon 12 of the DNMT1 gene in a young woman who presented with pure cerebellar ataxia. This report indicates that a mutation in exon 12 may lead to pure cerebellar ataxia. Another possibility is that the patient is currently in an early stage of the disease, and as the disease progresses, she will have more manifestations. To confirm or exclude this possibility, a subsequent follow-up study reporting the disease progression in this patient may be needed. Further reports of cases with the same mutation are needed to confirm the phenotype of this mutation.

\section{References}

1. Algahtani H, Marzouk Y, Algahtani R, Salman S, Shirah B. Autosomal recessive cerebellar ataxia type 1 mimicking multiple sclerosis: a report of two siblings with a novel mutation in SYNE1 gene in a Saud family. J Neurol Sci 2017;372:97-100.

2. Storey E. Genetic cerebellar ataxias. Semin Neurol 2014;34:280-92.

3. Baets J, Duan X, Wu Y, Smith G, Seeley WW, Mademan I, et al. Defects of mutant DNMT1 are linked to a spectrum of neurological disorders. Brain 2015;138:845-61.

4. Yuan J, Higuchi $Y$, Nagado $T$, Nozuma $S$, Nakamura $T$, Matsuura E, et al. Novel mutation in the replication focus targeting sequence domain of DNMT1 causes hereditary sensory and autonomic neuropathy IE. J Peripher Nerv Syst 2013;18:89-93.

5. Maresca A, Zaffagnini M, Caporali L, Carelli V, Zanna C. DNA methyltransferase 1 mutations and mitochondrial pathology: is mtDNA methylated? Front Genet 2015;6:90.

6. Feng J, Fan G. The role of DNA methylation in the central nervous system and neuropsychiatric disorders. Int Rev Neurobiol 2009;89:67-84.

7. Smets $M$, Link $S$, Wolf $P$, Schneider $K_{1}$ Solis $V_{1}$ Ryan J, et al. DNMT1 mutations found in HSANIE patients affect interaction with UHRF1 and neuronal differentiation. Hum Mol Genet 2017;26:1522-34.

8. Kernohan KD, Cigana Schenkel L, Huang L, Smith A, Pare G, Ainsworth $P$, et al. Identification of a methylation profile for DNMT1-associated autosomal dominant cerebellar ataxia, deafness, and narcolepsy. Clin Epigenetics 2016;8:91.

9. Winkelmann J, Lin L, Schormair B, Kornum BR, Faraco J, Plazzi G, et al. Mutations in DNMT1 cause autosomal dominant cerebellar ataxia, deafness and narcolepsy. Hum Mol Genet 2012;21:2205-10.

10. Klein CJ, Botuyan MV, Wu Y, Ward CJ, Nicholson GA, Hammans S, et al. Mutations in DNMT1 cause hereditary sensory neuropathy with dementia and hearing loss. Nat Genet 2011;43:595-600.

11. Klein CJ. DNMT1-related dementia, deafness, and sensory neuropathy. In: Adam MP, Ardinger HH, Pagon RA, Wallace SE, Bean $\sqcup H_{\text {, }}$ Mefford HC, eds. GeneReviews ${ }^{\circledR}$ [Internet]. Seattle (WA): University of Washington, 2012 Feb 16 [updated 2012 May 17; cited 2017 Jun 1] [https://www.ncbi.n/m.nih.gov/pubmed/22338191]. 